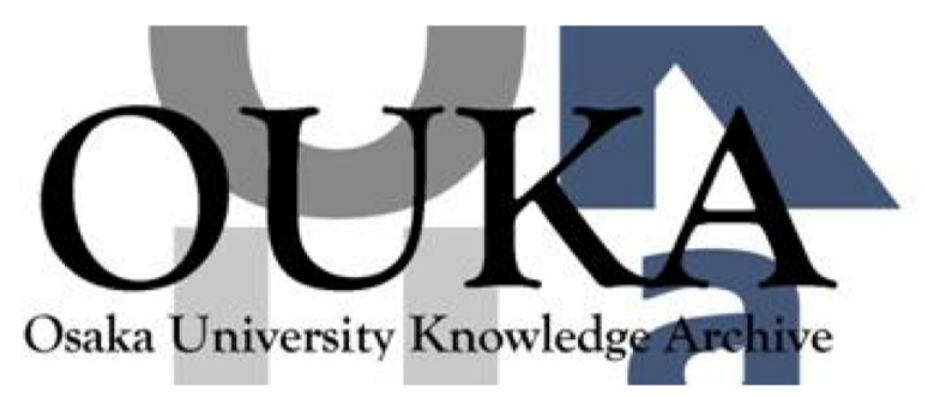

\begin{tabular}{|c|l|}
\hline Title & $\begin{array}{l}\text { Measurements of high energy density electrons } \\
\text { via observation of Cherenkov radiation }\end{array}$ \\
\hline Author(s) & $\begin{array}{l}\text { Habara, Hideaki; Ohta, Kazuhide; Tanaka, Kazuo } \\
\text { A. et al. }\end{array}$ \\
\hline Citation & Physics of Plasmas. 17(5) p.056306 \\
\hline Issue Date & $2010-03$ \\
\hline oaire:version & VoR \\
\hline URL & https://hdl. handle.net/11094/2999 \\
\hline rights & \\
\hline Note & \\
\hline
\end{tabular}

Osaka University Knowledge Archive : OUKA

https://ir. Library. osaka-u. ac. jp/

Osaka University 


\title{
Measurements of high energy density electrons via observation of Cherenkov radiation ${ }^{\text {a) }}$
}

\author{
Hideaki Habara, ${ }^{1, b)}$ Kazuhide Ohta, ${ }^{1}$ Kazuo A. Tanaka, ${ }^{1}$ G. Ravindra Kumar, ${ }^{2}$ \\ M. Krishnamurthy, ${ }^{2}$ Subhendu Kahaly, ${ }^{2, c)}$ Sudipta Mondal, ${ }^{2}$ Manoj Kumar Bhuyan, ${ }^{2}$ \\ R. Rajeev, ${ }^{2}$ and Jian Zheng ${ }^{3}$ \\ ${ }^{1}$ Graduate School of Engineering, Osaka University, 2-1, Yamada-oka, Suita, Osaka 565-0871, Japan \\ and Laser Institute of Engineering, Osaka University, 2-6, Yamada-oka, Suita, Osaka 565-0871, Japan \\ ${ }^{2}$ Tata Institute of Fundamental Research, Homi Bhabha Road, Mumbai 400-005, India \\ ${ }^{3}$ Department of Modern Physics, University of Science and Technology of China, Hefei, Anhui 230026, \\ People's Republic of China
}

(Received 20 November 2009; accepted 9 February 2010; published online 29 March 2010)

\begin{abstract}
Direct measurement of extremely high energy density electrons created in ultraintense laser-plasma interactions is crucial issue for fast ignition. Recently Cherenkov radiation has been studied to obtain the energy distribution of electrons because the emission angle depends on the electron energy. However in the previous studies [F. Brandl et al., Europhys. Lett. 61, 632 (2003); M. Manclossi et al., Phys. Rev. Lett. 96, 125002 (2006)], the experimental configurations using a planar target raised issues of spatial overlapping among the light from the different energy electrons as well as from the other emissions, such as transition radiation. A novel prism shaped target is developed in which Cherenkov lights emitted from different energy electrons are spatially separated, realizing an absolute measurement of the energy spectrum by counting the light intensities in each observed position. The observed image clearly shows the horseshoe pattern as expected in fully three-dimensional ray-trace calculations, and the image is successfully converted into the electron spectrum inside the target. In addition, it is found from the blur of the outer edge of the Cherenkov pattern that the electrons have a small beam divergence. The calibrated energy spectrum well agrees with particle simulations. (C) 2010 American Institute of Physics. [doi:10.1063/1.3346370]
\end{abstract}

\section{INTRODUCTION}

Production of extremely high energy density electrons created in ultraintense laser (UIL) plasma interactions promises exciting applications such as fast ignition (FI). ${ }^{1}$ In FI, because observations of absolute energy distribution of these electrons are definitely required for estimation of the heating efficiency to the core plasma, many theoretical and experimental contributions have been performed; for example, electron magnet spectrometer (ESM) has been successfully used for the absolute measurement of fast electrons escaping from the target. ${ }^{2}$ However the existence of giant self-excited electric and magnetic fields at target boundaries modifies the energy of escaping electrons by pulling back the electrons into the $\operatorname{target}^{3}$ so that the energy spectrum taken with ESM needs careful treatment.

Apart from the measurement of vacuum electrons, investigations have studied photons, from optical to $\mathrm{x}$-ray region, emitted via interactions of such energetic electrons with plasmas for estimation of the number of accelerated electrons inside the target. ${ }^{4-6}$ In particular, the Cherenkov radiation is studied to obtain the energy distribution of electrons because the emission angle of the Cherenkov light depends on the electron energy. However in the previous studies, the experimental configurations using a planar target have some prob-

\footnotetext{
a) Paper XI2 3, Bull. Am. Phys. Soc. 54, 343 (2009).

${ }^{b)}$ Invited speaker. Electronic mail: habara@eei.eng.osaka-u.ac.jp.

${ }^{c}$ Present address: Laboratoire d'Optique Applique, Ecole Polytechnique,

ENSTA, CNRS, UMR 7639, 91761 Palaiseau, France.
}

lems, for example, overlapping of the light from other radiations such as transition radiation and bremsstrahlung.

In order to overcome such difficulty, we developed a prism shaped target to obtain the energy distribution of fast electrons. ${ }^{7}$ The Cherenkov radiation emitted from different energy electrons is spatially separated in prism as well as the other emissions simultaneously, which realize an absolute measurement of the energy spectrum from the light intensities in each position. In Sec. II, basic properties of the Cherenkov radiation are introduced as well as the experimental results using a plane target. In Sec. III the development of prism shaped target is explained. Section IV describes the ray-trace calculation conducted for analysis of the observed Cherenkov image. Also several problems to be taken in accounts are addressed. Section V shows the preliminary experimental results and discussions. Then we summarize the results in Sec. VI.

\section{CHERENKOV RADIATION IN LASER PLASMA INTERACTIONS}

When a charged particle is passing through a dielectric material, the Cherenkov radiation can be emitted inside the material when the particle velocity exceeds the speed of light in the material, $c / n$, where $c$ is the speed of light in vacuum and $n$ is the refractive index of the material. The Cherenkov radiation has unique features that the emission angle and efficiency depend on the energy of the particle, given by 


$$
\cos \theta=\frac{1}{n(\lambda) \beta},
$$

where $\theta$ is the emission angle, $n(\lambda)$ is the refractive index as a function of emitted light wavelength $\lambda$, and $\beta=v / c$, and

$$
\frac{\mathrm{d} N}{\mathrm{~d} \lambda}=\frac{2 \pi \alpha L}{\lambda^{2}}\left(1-\frac{1}{n(\lambda)^{2} \beta^{2}}\right),
$$

where $N$ is the number of emitted photons, $\alpha$ is the fine structure constant, and $L$ is the propagation length of particle. ${ }^{8,9}$

From these features, the observation of the Cherenkov radiation is now one of common techniques in high energy particle physics. For example, threshold energy to emit the Cherenkov light is used to identify the species of particle and the emission angle is measured to obtain the particle energy. $^{10}$

The measurement of the Cherenkov radiation has been also applied to obtain the energy spectrum of electrons generated in UIL-plasma interactions. In the previous experiments, an intense laser beam irradiates a thin metal foil. Then the generated electrons propagate in the glass plate immediately behind the foil and emit the Cherenkov light. Brandl et al. ${ }^{11}$ observed the Cherenkov image far from the glass plate via collection lens onto the gated charge coupled device (CCD). In order to obtain the energy distribution of fast electrons, they carried out GEANT Monte Carlo calculations to reproduce the experimental image. Their analysis reveals that there are two populations of electrons with different propagation directions, energies, and divergence angles, as already showed by Santala et al. ${ }^{12}$ Manclossi et al. ${ }^{13}$ confirmed the Cherenkov emission from laser produced electrons via their observations that the Cherenkov light intensities increase with the thickness of dielectric material. However as in the experiments above, if the Cherenkov light is observed apart from the target, some portion of Cherenkov light is totally reflected at the dielectric-vacuum boundary. Higher energy electrons emit the Cherenkov light with larger angles so that in the case of BK7 $(n=1.516$ at $600 \mathrm{~nm})$, the Cherenkov radiation emitted from fast electrons whose energies are over $0.55 \mathrm{MeV}$ cannot be observed. It is a significant problem for observation of total energy distribution of the fast electrons. Moreover, diffraction at the rear surface complicates the analysis of the emission angle of the Cherenkov radiation. In order to observe the whole pattern with the large angle Cherenkov radiations, we performed an experiment using a planar target whose rear surface becomes frosted to observe the scattered light at the rear surface.

\section{A. Experiment using a plane target}

The experiment is performed using a Ti:sapphire $10 \mathrm{TW}$ laser system at Tata Institute of Fundamental Research (TIFR), India. The typical laser energy on target is about $260 \mathrm{~mJ}$ operated in $10 \mathrm{~Hz}$. The pulse duration is close to the Fourier limit of 30 fs with nano- and picosecond contrasts of $10^{5}$ and $10^{6}$, respectively. The laser pulse is focused via $\mathrm{f} 3$ off-axis parabolic mirror to $10 \mu \mathrm{m}$ diameter, resulting in the laser intensity of $8.5 \times 10^{18} \mathrm{~W} / \mathrm{cm}^{2}$. The laser hits the target
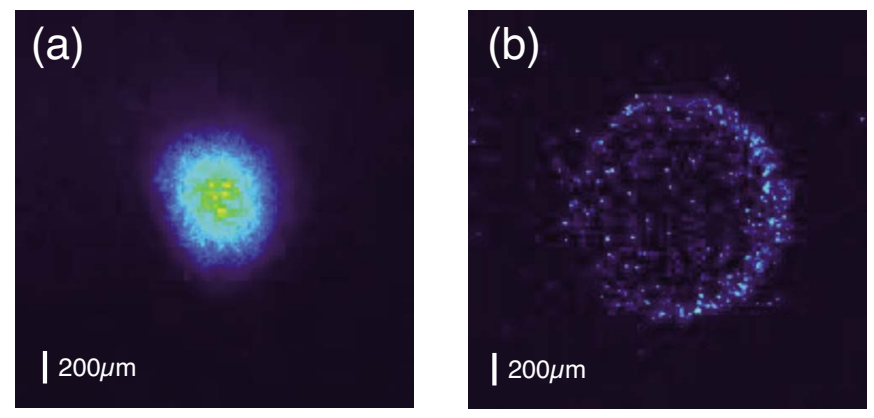

FIG. 1. (Color online) Typical plane target experimental image for (a) double and (b) single layer targets.

at near normal incidence $\left(\sim 5^{\circ} p\right.$-polarization). The target consists of front $\mathrm{Al}$ layer $(11 \mu \mathrm{m}$ thickness $)$ and fused quartz $(n=1.52$ at $600 \mathrm{~nm})$. The thickness of quartz is changed from 50 to $100 \mu \mathrm{m}$.

The Cherenkov image appearing on the rear surface is observed with an intensified charged-coupled device (ICCD) camera at $42^{\circ}$ from the laser axis. The image is collected via two achromatic lenses with the magnification of near 1 . Although the Cherenkov light is usually measured in the violet wavelength with narrow bandpass filter where the emission intensity becomes strongest, a longer wavelength was used in this case. Because the refractive index is relatively constant in this region, we could use a wider bandwidth, 575-750 nm, without blurring the image to compensate for its lower intensity, nearly $1 / 3$ of the peak in the violet. We used the bandpass filter with $80 \%$ flattop transmittance.

Figure 1(a) shows a typical experimental image taken from a double layered target with $50 \mu \mathrm{m}$ thickness of fused quartz. For comparison, the image from a single layer target with similar thickness (50 $\mu \mathrm{m} \mathrm{Al})$ is also shown in Fig. 1(b). The observation conditions, such as gain of ICCD camera, gated temporal window ( $\sim 5 \mathrm{~ns})$, filters, and so on, are fixed through the experiment. Because the single foil does not contain the dielectric material, the image from the single foil may indicate purely transition radiations emitted at the rear boundary of the target. On the other hand, the observed image at double layered target may include both the Cherenkov radiation and transition radiation emitted not only at the target rear surface but also at the metal-dielectric boundary. Comparing Figs. 1(a) and 1(b), the spot size at single layer is about two times larger than that at double layer in spite of nearly same target thickness. Figure 2(a) shows the summary of the spot size in $1 / e^{2}$ for double (circles) and single (squares) layer targets as a function of target thickness. In the case of transition radiation, spot diameter is not proportional to the target thickness probably due to reduction in beam density by scattering of the lower energy electrons. On the other hand, the spot diameter on double layer target seems to increase with the target thickness. Assuming that the emissions are mainly contributed by the Cherenkov radiation in order to simplify the discussion, the emission angle of the electron beam is estimated to be $\pm 24^{\circ}$ by substituting the maximum emission angle of the Cherenkov radiation $\left(\sim 45^{\circ}\right)$ into the slope of the fitting curve. This angle is rela- 

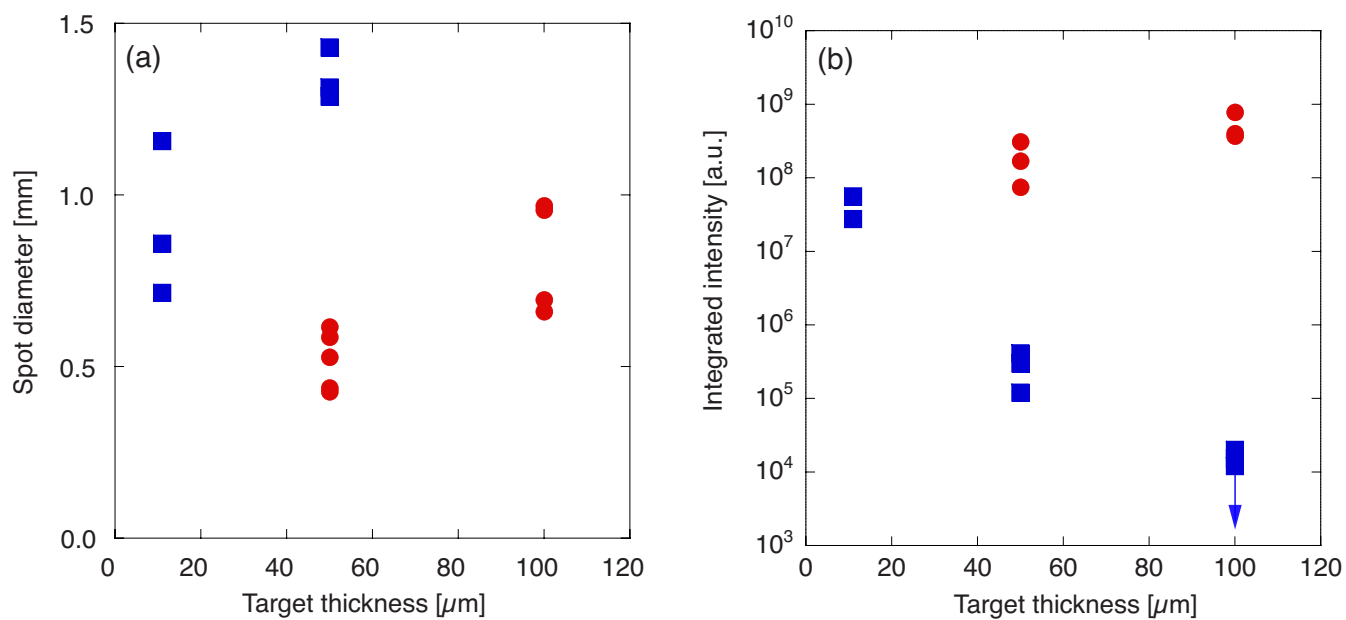

FIG. 2. (Color online) (a) Spot diameter and (b) integrated signal intensities as a function of target thickness of plane target. The closed circles and squares are data from double and single layer targets, respectively.

tively close to the results obtained in Ref. 11. Note that the stopping range of $200 \mathrm{keV}$ electrons is enough longer $(\sim 150 \mu \mathrm{m})$ than the target thickness.

In addition, the integrated signal intensity from double layer target in Fig. 1(a) is 100 times larger than that of transition radiation in Fig. 1(b). Figure 2(b) shows signal intensities as a function of target thickness. The dots and squares represent the integrated intensities taken from the double and single layered targets, respectively. The signal intensities are accumulated over the $1 / e^{2}$ diameter in the spot. Apparently, the light intensity from the double layer target increases with the target thickness. This might be an evidence that the emission at the double layer target is dominated by the Cherenkov radiation because the intensity of Cherenkov photons increases with the propagation length of electrons in the dielectric target. On the other hand, the light intensity of transition radiation seems to rapidly decrease with the target thickness as the same reason with the reduction of spot size. In particular, the difference in intensities between single and double layered targets well agrees with the previous plane target experiment by Manclossi et al. ${ }^{13}$

\section{PRISM SHAPED TARGET}

Measurement of Cherenkov radiations using a planar target gives us rough estimations of the energy distribution and beam divergence. However, these accuracies are just similar to the other experimental results from the observation of $\mathrm{K} \alpha$ $\mathrm{x}$ rays ${ }^{4}$ or transition radiation. ${ }^{5}$ In addition the results using the planar target include several problems on the interpretation of the observed image. One is the overlapping of other radiations such as transition radiation and bremsstrahlung on the Cherenkov image.

On the other hand, the total reflection and diffraction are also problems as mentioned above. Although this can be avoided when the rear surface becomes frosted as in our target, the Cherenkov light emitted from different energy electrons are totally overlapped at the rear surface because the Cherenkov radiation is continuously emitted during the propagation of electrons in the dielectric material. This causes problems in resolving the electron energy only from the detected image without assumption on the shape of energy spectrum.

In order to overcome these difficulties, we developed a prism shaped target, as shown in Fig. 3. In the figure, the Cherenkov lights emitted by higher (right), medium (center), lower (left) energy electrons (as shown by the circles in the right side in the figure) are reflected at the rear surface of the prism target and are then delivered into the frosted bottom surface at spatially different positions. Therefore when the light intensities are measured at the bottom surface, the electron energy distribution is easily obtained from the Cherenkov pattern directly.

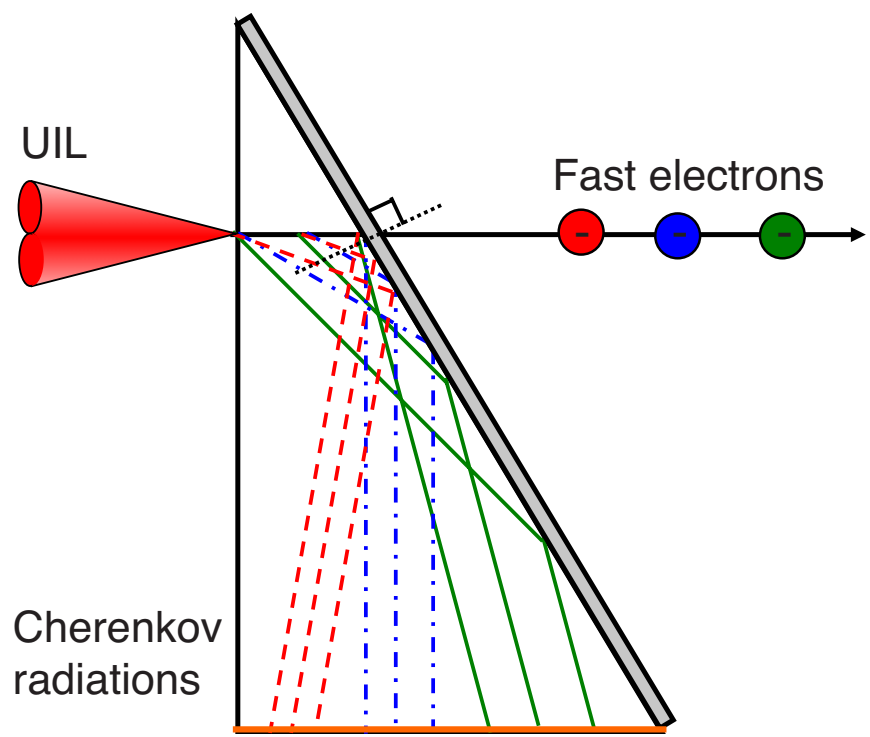

FIG. 3. (Color online) Schematic image of the Cherenkov radiation in prism target. The fast electrons generated by UIL light are represented by circles in the right side, corresponding to higher (right), medium (center), and lower (left) energies. Each electron emits the Cherenkov light during the propagation. The paths of these Cherenkov rays emitted from lower, medium, and higher energy electrons are represented by dashed, dashed-dotted, and solid lines, respectively. 

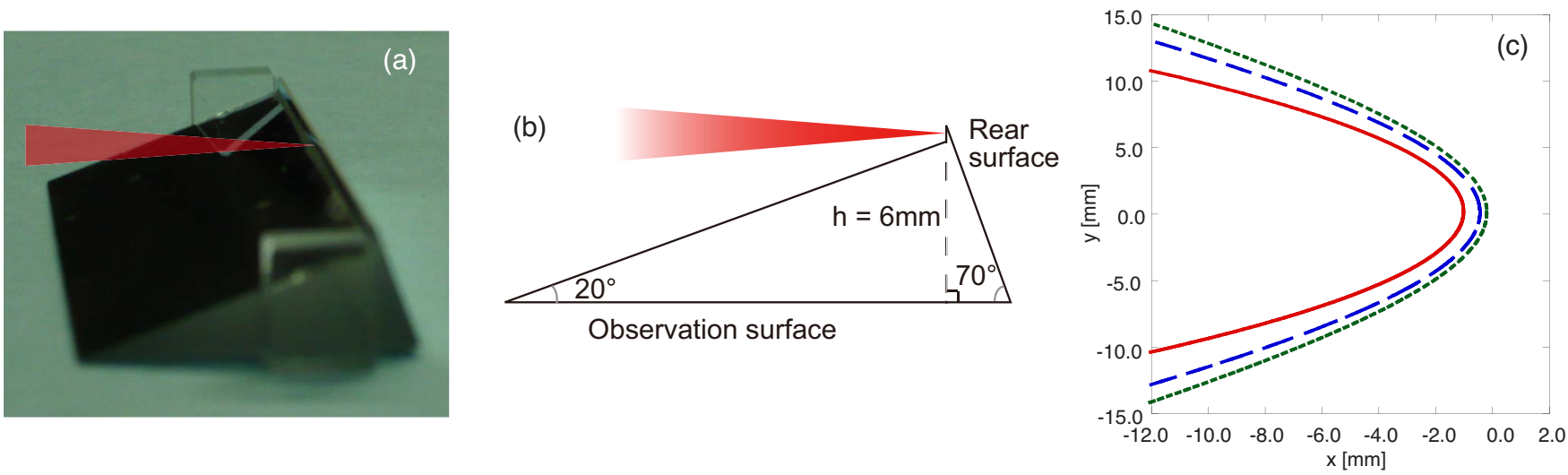

FIG. 4. (Color online) (a) Picture of actual prism target. The triangle from left to right in the figure represents the incident laser light. (b) Side view of the prism target. The target consists of two right angle prisms. The triangle also shows the laser direction. (c) Cherenkov light pattern at the bottom surface calculated by the ray-trace calculation. As same as (a), the laser direction is taken from left to right. The outer, center, and inner lines represent the Cherenkov light from 3.0, 1.0, and $0.5 \mathrm{MeV}$ electrons, respectively.

\section{RAY-TRACE MODELING}

We performed a three-dimensional (3D) ray-trace calculation in order to understand the possible Cherenkov image observed at the bottom surface of the prism target. Figure 4(a) shows a picture of prism target we used and (b) the actual dimensions that are taken into account in the calculations. This prism target consists of two right triangles, each of which smaller acute angle is $20^{\circ}$. The laser light, indicated by a triangle, irradiates the thin tip of the thinner prism upper edge. The Cherenkov light is reflected at the right tilting surface and then goes into the frosted bottom through the second prism. The height from the tip to the bottom is $6 \mathrm{~mm}$ and the area of bottom surface is $18 \times 44 \mathrm{~mm}^{2}$. The thickness of thin tip is $30 \mu \mathrm{m}$ in order to obtain enough intense Cherenkov light without significant beam scattering. In the result of calculation the observable image shows a horseshoe pattern. Figure 4(c) shows the Cherenkov light patterns emitted from 3.0 (dotted lines), 1.0 (dashed lines), and $0.5 \mathrm{MeV}$ (solid lines) electrons at the bottom surface, respectively. For the simplicity, the emission wavelength is fixed to $600 \mathrm{~nm}$. It is clear that the spatial dispersion of each Cherenkov light is largest for vertical directions so that the vertical line profile gives an energy distribution of fast electrons with highest energy resolution.

Figure 5 shows the calculation results of Cherenkov light at the rear surface of the planar target changing the electron energy distribution for (a) simple exponential decay and (c) a relativistic Maxwell distribution. In both cases, the slope temperatures are assumed as $1 \mathrm{MeV}$. Figures 5(b) and 5(d) represent the expanded image around outer edge of the pat-
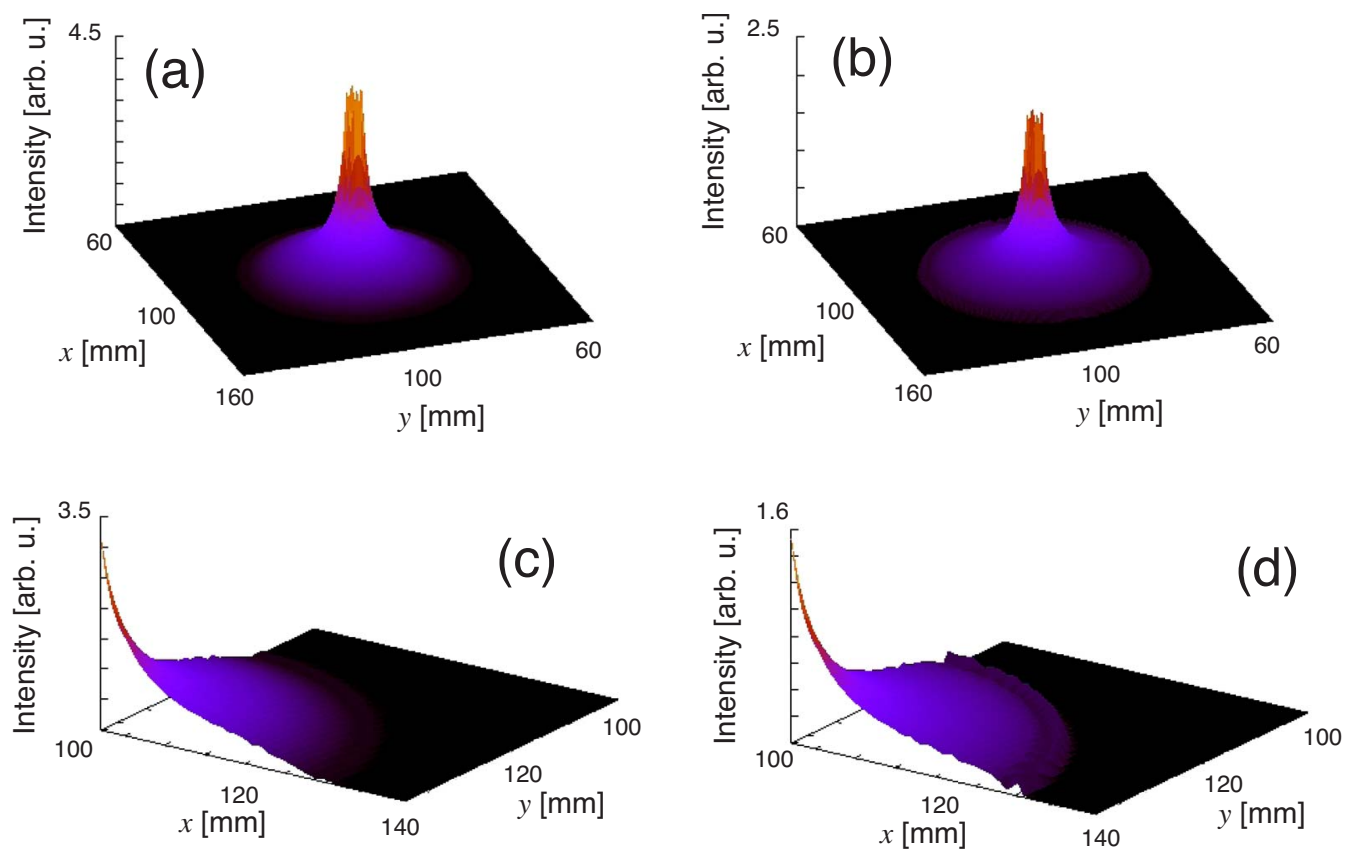

FIG. 5. (Color online) Whole image of the Cherenkov radiation pattern for plane target assuming the electron energy distribution of (a) exponential decay and (b) relativistic Maxwell distribution by ray-trace calculations. (c) and (d) show the expanded image around the outer edge of the whole pattern of (a) and (b), respectively. 

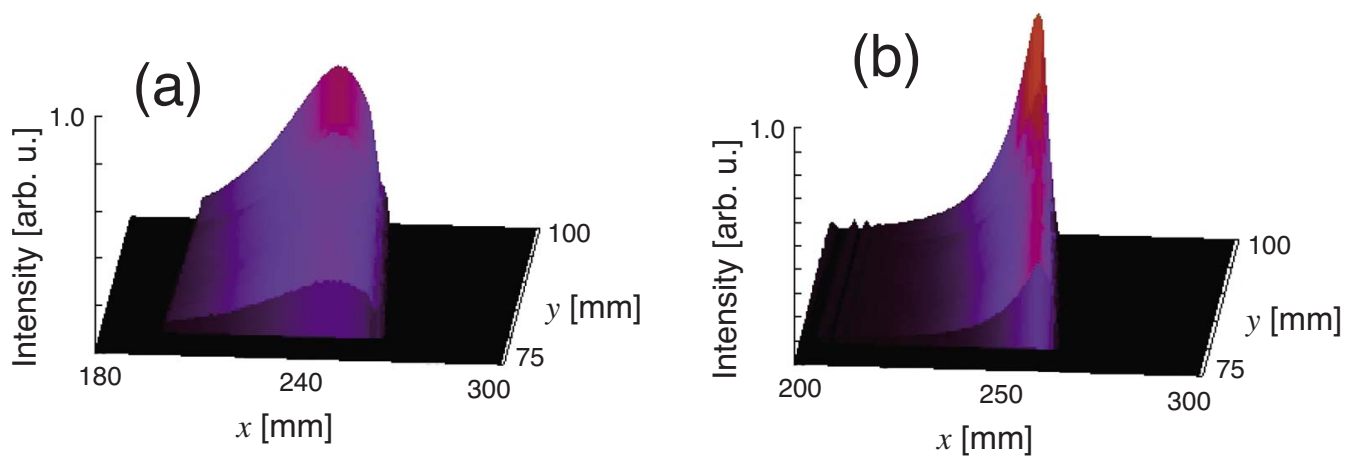

FIG. 6. (Color online) Expanded image around the one arm in the whole horseshoe pattern in bottom surface of prism target for (a) exponential decay and (b) relativistic Maxwell electron energy distribution.

tern of (a) and (c), respectively. For the planar target, there are almost no differences between these two energy distributions even in the expanded images. This fact implies that the observation of the Cherenkov image using a plane target cannot reconstruct the electron energy distribution exactly. On the other hand, Fig. 6 indicates the results from prism target for simple exponential decay and relativistic Maxwell distribution. Again, Figs. 6(a) and 6(b) represent the expanded image around the one arm of the horseshoe pattern in each case, respectively. The left side in the image corresponds to the Cherenkov light from lower energy electrons and the right side to the higher energy electrons. The expanded images indicate clearly different patterns on its cross section. From these results, the prism target can identify the electron energy distribution when the spatial resolution of detector is high enough. Note that the spatial resolution can easily increase with the height from interaction point to the bottom surface of the prism target.

\section{A. Beam divergence}

In the above ray-trace calculation we defined that electrons propagate straight into laser axis. However it is known that the fast electron beam has some amount of divergence from measurement of $\mathrm{K} \alpha$ emissions or transition radiation. It might be a significant problem for converting the electron energy distribution because of obscuring the Cherenkov pattern. Fortunately, from Eq. (1), emission angle of the Cherenkov light is saturated at the higher energy electrons because $\beta$ becomes relatively constant and close to 1 . For example, the emission angles are $23^{\circ}$ and $37^{\circ}$ for 0.5 and $1.0 \mathrm{MeV}$ electrons, respectively, whereas $46^{\circ}$ for $4 \mathrm{MeV}$ and $48^{\circ}$ for $10 \mathrm{MeV}$ electrons in the case of BK7. This feature makes a clear edge in the outer side of the Cherenkov pattern. In other words, the emission divergence can be estimated from the blur of the outer edge of the observed pattern. As shown in Fig. 4(b), the spatial dispersion of the Cherenkov light from different energy electrons becomes smallest for horizontal direction. Consequently the horizontal line profile gives the beam divergence of fast electrons. If the beam divergence has a dependence on electron energies, the analysis could be significantly difficult. However because the threshold energy of electron emitting the Cherenkov radia- tion is a function of $n$, the energy depended beam divergence can be estimated by using a different material having various refractive indexes.

\section{B. Target ionization}

Target ionization is a serious problem to estimate the number of fast electrons. The electrons generated in laserplasma interactions are so intense that the electrostatic field ahead of the beam can easily ionize the target. In the result, the Cherenkov light has never been emitted from the ionized part because the refractive index drops less than 1. Such ionized region in the target expands nearly $80 \%$ of the speed of light along the electron propagation direction from the previous observations, ${ }^{14,15}$ resulting that only small population of the electron beam being able to advance the ionization front speed can contribute to the Cherenkov radiation.

Recent numerical analyses indicate that the ionization speed is a function of beam density. ${ }^{16,17}$ In our experiment, electron beam density can be approximately $10^{19}-10^{20} \mathrm{~cm}^{-3}$ considering the energy conversion efficiency to be $30 \%-$ $40 \%$ from other experimental or simulation results. Assuming the energy distribution as Maxwellian with $1 \mathrm{MeV}$ slope temperature from the Ponderomotive scaling, ${ }^{18}$ the ionization front velocity will be $0.8 \mathrm{c}$ according to Ref. 17 . From this ionization front velocity, it can be calculated that nearly $14 \%$ of fast electrons in this energy distribution can emit the Cherenkov radiation. On the other hand, the observed Cherenkov light intensity gives the absolute number of this " $14 \%$ of electrons" so that total number of fast electrons, which corresponds to beam density, can be easily estimated. Then we can go back to the estimation of ionization front velocity from the references again. These iterative considerations enable us to reconstruct the ionization front speed and beam density simultaneously.

\section{PRISM TARGET EXPERIMENT}

We conducted an experiment using a prism target also at TIFR. The laser conditions are nearly same with those in plane target. The laser energy slightly increases to $280 \mathrm{~mJ}$ on target, resulting in the laser intensity of $9.2 \times 10^{18} \mathrm{~W} / \mathrm{cm}^{2}$. This laser is focused on the very thin tip of the prism target, as shown in Fig. 4(a) or Fig. 4(b). Two diagnostics are used 


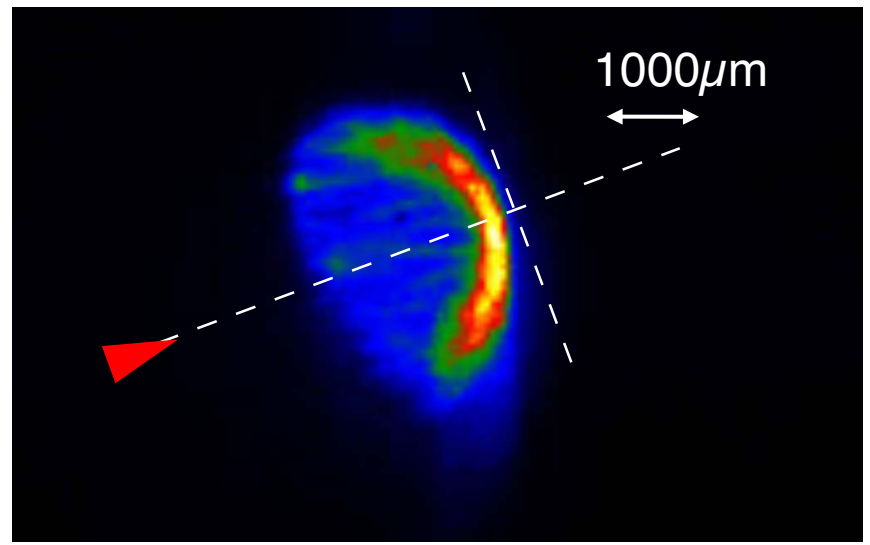

FIG. 7. (Color online) Typical observed image at the prism bottom surface. The laser direction is represented by the triangle at the left side of the figure.

for measurement of fast electrons; one is ESM for measurement of escaping electrons from the target. The ESM is placed in $55 \mathrm{~cm}$ behind the target on the laser axis. The detectable energy range of the electrons is between 0.1 and 7.3 MeV. Another detector is ICCD camera to observe the Cherenkov image at the bottom surface of the prism target. The similar optical setup is used with plane target experiment except with the magnification to be 0.22 in order to measure whole the Cherenkov image with $58 \mu \mathrm{m}$ spatial resolution, resulting in less than 5\% of energy resolution.

Figure 7 indicates a typical Cherenkov pattern taken at the bottom surface. The image shows clear horseshoe pattern as predicted by ray-trace calculations. For the analysis of beam divergence, the horizontal line profile is taken as shown by a solid line in Fig. 8. The ray-trace calculations are also plotted as the dashed or dotted lines in the figure. For the calculations, the input electron energy distribution is assumed to be a Maxwellian with different slope temperatures and several beam divergence angles. The calculation results indicate that the right part of the experimental line shape from the peak, corresponding to the outer edge of the Cherenkov pattern, is relatively independent to the slope temperature, but strongly depends on the beam divergence. The difference in slope temperature only appears on the left side slope from the peak when the temperature is less than $1 \mathrm{MeV}$. (If the temperature exceeds $1.5 \mathrm{MeV}$, the left slope does not changed so much.) In the result, $\pm 5^{\circ}$ beam divergence with $1.5-5.0 \mathrm{MeV}$ slope temperature is best fit to the experimental result. It should be noted that this straight propagation of fast electrons in the dielectric material have been already observed in the previous channel formation experiments inside a glass plate at different groups. ${ }^{14,15}$

Compared to the results from the planar target experiment, the beam divergence is completely different although the laser condition is almost same. However, as it has been pointed out in Sec. III, this discrepancy might be caused by overlapping of the transition radiation emitted at the metalquartz boundary. According to Eq. (2), about 0.48 photons are emitted from one $1 \mathrm{MeV}$ electron with $50 \mu \mathrm{m}$ propagation length. This is about 100 times stronger than that of transition radiation as in the Appendix. However the population of lower energy electrons is much larger than the higher

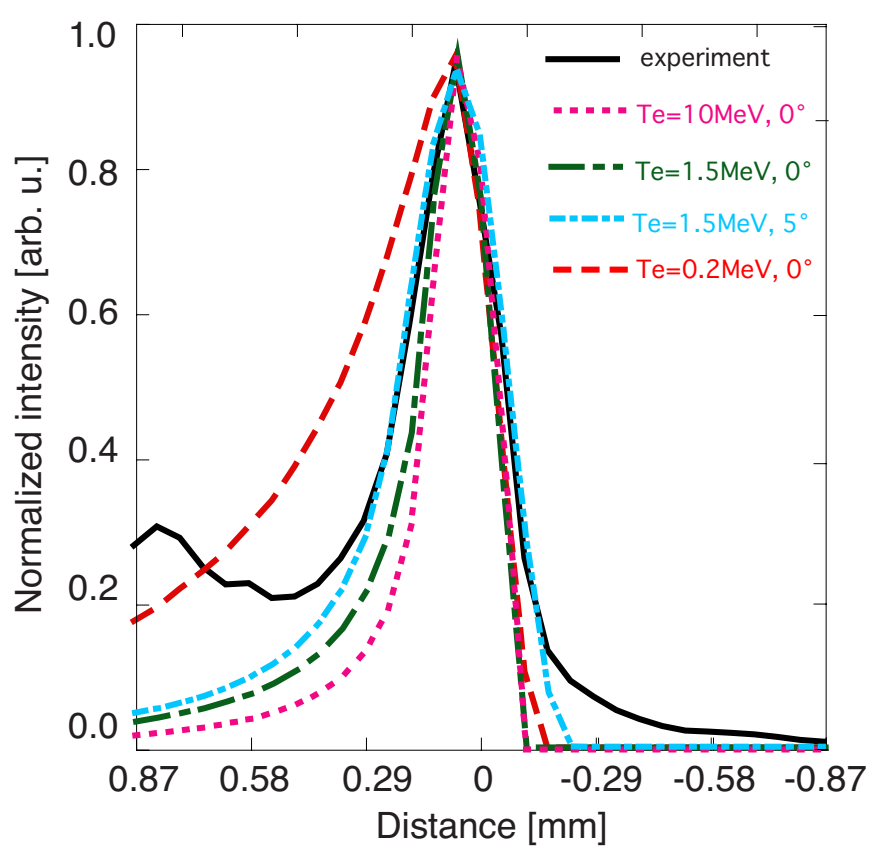

FIG. 8. (Color online) Horizontal line shapes. Solid line is taken from Fig. 7. Other lines are the ray-trace calculations assuming Maxwell energy distributions with $0.2 \mathrm{MeV}$ (dashed line), 1.5 MeV (longer dashed-dotted line), and $10 \mathrm{MeV}$ (dotted line) of slope temperature and no beam divergence, respectively. The shorter dashed-dotted lines are calculation for $1.5 \mathrm{MeV}$ temperature with $10^{\circ}$ divergence.

energy electrons emitting the Cherenkov radiation. This consideration agrees with the fact that the integrated intensities of transition radiation from $11 \mu \mathrm{m} \mathrm{Al}$ are just nearly half of the Cherenkov intensities from $50 \mu \mathrm{m}$ quartz target. In addition the emission angle of transition radiation emitted from lower energy electrons can be expected to be close to hemisphere direction, ${ }^{5}$ which might lead to the apparent larger divergence angle, as in the spot size of transition radiation in Fig. 2(a). Moreover, because the propagation length of plane target is longer than the prism target, beam scattering could broaden the apparent spot size of the Cherenkov pattern. Figure 9 indicates the experimental line profile (solid line) at the rear surface of $100 \mu \mathrm{m}$ quartz target with the calculated results from $3 \mathrm{D}$ ray-trace calculations assuming $\pm 5^{\circ}$ (dashed line) and $\pm 30^{\circ}$ (dashed-dotted line) beam divergences with $500 \mathrm{keV}$ exponential electron energy distribution. The difference of spot sizes in both calculations and the experiment are just within $10 \%$ (corresponds to $2-3$ pixels on CCD in our experimental condition). Because the peak around $0 \mu \mathrm{m}$ would decrease due to beam scattering, which is not included in the calculation, both calculated line profiles become more close to the experiment. From these facts, not only the energy spectrum but also the exact beam divergence would be difficult to obtain from Cherenkov radiation using a plane target.

On the other hand, we can obtain the energy spectrum from the vertical line profile of Fig. 7. From the iterative analysis discussed in Sec. IV B, the beam density and ionization front are about $10^{19} \mathrm{~cm}^{-3}$ and $0.83 \mathrm{c}$. In the result, the reconstructed energy distribution from the Cherenkov measurement is given by closed circles in Fig. 10. The electron 


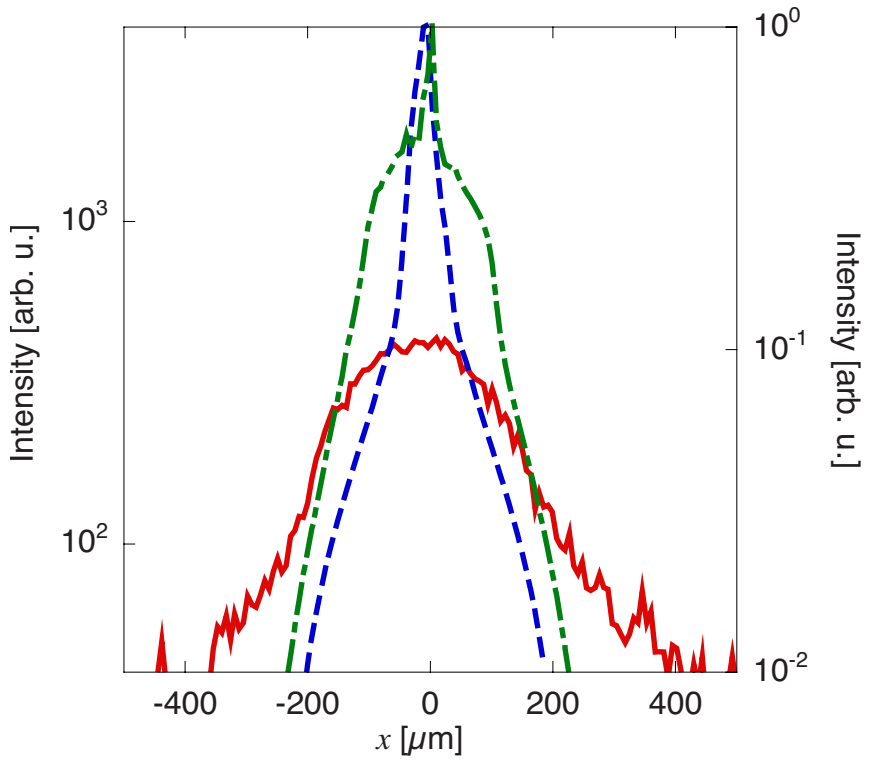

FIG. 9. (Color online) Line profiles of Cherenkov light on plane target. The solid line is the experimental results. Dashed and dashed-dotted lines are results from fully ray-trace calculations with $\pm 5^{\circ}$ and $\pm 30^{\circ}$ beam divergence with $500 \mathrm{keV}$ slope temperature of simple exponential decay electron energy distribution.

energy spectra taken from ESM are shown by open squares. Also the spectrum from a one-dimensional (1D)-PIC calculation is indicated by closed triangles. The calculation conditions such as laser intensities and preformed plasma scale are taken from the exact experimental parameters. Clearly this calibrated spectrum well agrees with the 1D-PIC calculation rather than the ESM spectrum. In addition, it is interesting to point out that the slope of the Cherenkov spectrum seems similar with that of ESM up to $200 \mathrm{keV}$. (The ESM spectrum over $200 \mathrm{keV}$ is close to noise level.) This fact might indicate the energy Doppler shift by the sheath potential at the rear side of the target, ${ }^{3}$ which causes electron refluxing ${ }^{19}$ and ion acceleration by target normal sheath acceleration mechanism. $^{20}$

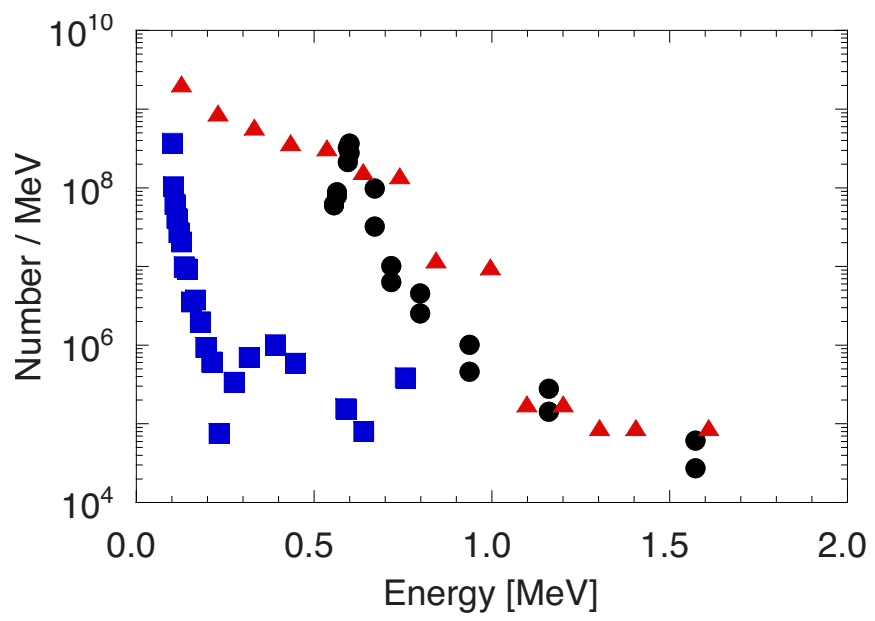

FIG. 10. (Color online) Electron energy distributions from ESM (solid squares), Cherenkov image (closed circles), and 1D PIC calculation (solid triangles), respectively.

\section{SUMMARY}

The Cherenkov radiation enables us to observe fast electrons propagating inside the dielectric target directly. Prism target can resolve the energy distribution of electrons by spatially resolving the Cherenkov light. Although our planar target experiments well reproduced the previous Cherenkov experiment regarding to the beam divergence and difference of emission intensities to the transition radiation, the prism target experiment reveals that these interpretations might lead to misunderstanding. The analysis of the Cherenkov light image in prism target experiment indicates the low beam divergence, and shows the energy distribution well agrees with the predictions with the PIC calculations.

\section{ACKNOWLEDGMENTS}

This work was fully supported by international collaboration program of Japan Society for the Promotion of Science (JSPS) and the Indian National Science Academy (INSA). A part of instruments was supported by Grant-inAid for Scientific Research of JSPS (Contract No. 19206099). G.R.K. acknowledges an ORI grant from the DAE-SRC, Government of India. A part of the research was also supported by the JSPS-CUP (Core University Program) in fusion area operated by NIFS (National Institute of Fusion and Science). The authors gratefully acknowledge Professor H. Sakagami, National Institute for Fusion Science, Japan, for his permission to use his 1D-PIC code.

\section{APPENDIX: EMISSION INTENSITY OF TRANSITION RADIATION}

According to Ref. 8, the emission intensity $(I)$ of the transition radiation is presented by

$$
\begin{aligned}
\frac{\mathrm{d}^{2} I}{\mathrm{~d} \nu \mathrm{d} \eta} & =\frac{\pi}{\gamma^{2}} \gamma \omega_{p} \frac{\mathrm{d}^{2} I}{\mathrm{~d} \omega} \\
& \simeq \frac{e^{2} \gamma \omega_{p}}{\pi c}\left[\frac{\eta}{\nu^{4}\left(1+1 / \nu^{2}+\eta\right)^{2}\left(1+\eta^{2}\right)}\right],
\end{aligned}
$$

where $\nu$ is the normalized frequency given by $\nu=\omega / \gamma \omega_{p}(\omega$ and $\omega_{p}$ are the emitted light and plasma frequency), $\eta$ the normalized emission angle given by $(\gamma \theta)^{2}$, and $\Omega$ the solid angle $(\mathrm{d} \phi \mathrm{d} \cos \theta)$. Integrated over the normalized frequency and the emission angles, the intensity of the tradition radiation generated by single electron is given by

$$
I=\frac{1}{3} \alpha \gamma \hbar \omega_{p},
$$

where $\alpha$ is the fine structure constant. If the electron energy is $1 \mathrm{MeV}$, the emission energy becomes $1.34 \times 10^{-21} \mathrm{~J}$. Here, the photon energy for $600 \mathrm{~nm}$ light is $3.3 \times 10^{-19} \mathrm{~J}$. It is known that the nearly half of the emission intensity of the transition radiation ranges between $0.1<\nu<1$ (corresponding to $358-3580 \mathrm{~nm}$ ) so that the maximum number of photons becomes $4 \times 10^{-3}$ per electron assuming that the half of the total intensity is in our observation wavelength.

\footnotetext{
${ }^{1}$ M. Tabak, J. Hammer, M. E. Glinsky, W. L. Kruer, S. C. Wilks, J. Woodworth, E. M. Campbell, M. D. Perry, and R. J. Mason, Phys. Plasmas 1, 1626 (1994).
} 
${ }^{2}$ K. A. Tanaka, T. Yabuuchi, T. Sato, R. Kodama, Y. Kitagawa, T. Takahashi, T. Ikeda, Y. Honda, and S. Okuda, Rev. Sci. Instrum. 76, 013507 (2005).

${ }^{3}$ T. Yabuuchi, K. Adumi, H. Habara, R. Kodama, K. Kondo, T. Tanimoto, K. A. Tanaka, Y. Sentoku, T. Matsuoka, Z. L. Chen, M. Tampo, A. L. Lei, and K. Mima, Phys. Plasmas 14, 040706 (2007).

${ }^{4}$ R. B. Stephens, R. A. Snavely, Y. Aglitskiy, F. Amiranoff, C. Andersen, D. Batani, S. D. Baton, T. Cowan, R. R. Freeman, T. Hall, S. P. Hatchett, J. M. Hill, M. H. Key, J. A. King, J. A. Koch, M. Koenig, A. J. MacKinnon, K. L. Lancaster, E. Martinolli, P. Norreys, E. Perelli-Cippo, M. Rabec Le Gloahec, C. Rousseaux, J. J. Santos, and F. Scianitti, Phys. Rev. E 69, 066414 (2004).

${ }^{5}$ J. Zheng, K. A. Tanaka, T. Miyakoshi, Y. Kitagawa, R. Kodama, T. Kurahashi, and T. Yamanaka, Phys. Plasmas 9, 3610 (2002); Phys. Rev. Lett. 92, 165001 (2004).

${ }^{6}$ M. Nakatsutsumi, J. R. Davies, R. Kodama, J. S. Green, K. L. Lancaster, K. U. Akli, F. N. Beg, S. N. Chen, D. Clark, R. R. Freeman, C. D. Gregory, H. Habara, R. Heathcote, D. S. Hey, K. Highbarger, P. Jaanimagi, M. H. Key, K. Krushelnick, T. Ma, A. MacPhee, A. J. MacKinnon, H. Nakamura, R. B. Stephens, M. Storm, M. Tampo, W. Theobald, L. Van Woerkom, R. L. Weber, M. S. Wei, N. C. Woolsey, and P. A. Norreys, New J. Phys. 10, 043046 (2008).

${ }^{7}$ H. Habara, K. Ohta, K. A. Tanaka, G. R. Kumar, M. Krishnamurthy, S. Kahaly, S. Mondal, M. K. Bhuyan, R. Rajeev, and J. Zheng, Phys. Rev. Lett. 104, 055001 (2010).

${ }^{8}$ J. D. Jackson, Classical Electrodynamics, 2nd ed. (Wiley, New York, 1975).

${ }^{9}$ J. Zheng, C. X. Yu, Z. J. Zheng, and K. A. Tanaka, Phys. Plasmas 12, 093105 (2005).
${ }^{10}$ G. F. Knoll, Radiation Detection and Measurement, 3rd ed. (Wiley, New York, 2000).

${ }^{11}$ F. Brandl, G. Pretzler, D. Habs, and E. Fill, Europhys. Lett. 61, 632 (2003).

${ }^{12}$ M. I. Santala, M. Zepf, I. Watts, F. N. Beg, E. Clark, M. Tatarakis, K. Krushelnick, A. E. Dangor, T. McCanny, I. Spencer, R. P. Singhal, K. W. Ledingham, S. C. Wilks, A. C. Machacek, J. S. Wark, R. Allott, R. J. Clarke, and P. A. Norreys, Phys. Rev. Lett. 84, 1459 (2000).

${ }^{13}$ M. Manclossi, J. J. Santos, D. Batani, J. Faure, A. Debayle, V. T. Tikhonchuk, and V. Malka, Phys. Rev. Lett. 96, 125002 (2006).

${ }^{14}$ L. Gremillet, F. Amiranoff, S. D. Baton, J.-C. Gauthier, and M. Koenig, Phys. Rev. Lett. 83, 5015 (1999).

${ }^{15}$ M. Borghesi, A. J. Mackinnon, A. R. Bell, G. Malka, C. Vickers, O. Willi, J. R. Davies, A. Pukhov, and J. Meyer-ter-Vehn, Phys. Rev. Lett. 83, 4309 (1999).

${ }^{16}$ S. I. Krasheninnikov, A. V. Kim, B. K. Frolov, and R. Stephens, Phys. Plasmas 12, 073105 (2005).

${ }^{17}$ O. Klimo, V. T. Tikhonchuk, and A. Debayle, Phys. Rev. E 75, 016403 (2007).

${ }^{18}$ S. C. Wilks, W. L. Kruer, M. Tabak, and A. B. Langdon, Phys. Rev. Lett. 69, 1383 (1992).

${ }^{19}$ Y. Sentoku, T. E. Cowan, A. Kemp, and H. Ruhl, Phys. Plasmas 10, 2009 (2003).

${ }^{20}$ S P. Hatchett, C. G. Brown, T. E. Cowan, E. A. Henry, J. S. Johnson, M. H. Key, J. A. Koch, A. Bruce Langdon, B. F. Lasinski, R. W. Lee, A. J. Mackinnon, D. M. Pennington, M. D. Perry, T. W. Phillips, M. Roth, T. C. Sangster, M. S. Singh, R. A. Snavely, M. A. Stoyer, S. C. Wilks, and K. Yasuike, Phys. Plasmas 7, 2076 (2000) 